\title{
Combined thoracic paravertebral and PECS 2 block as an alternative to general anaesthesia for major breast surgery - A case report
}

\author{
K. Duraisamy, V. Woodham, A. Pawa. \\ Guy's and St.Thomas NHS Foundation trust, London, United Kingdom
}

\section{Background and aims:}

Breast cancer surgery is associated with a significant amount of intraoperative and postoperative pain and is a key risk factor for the development of chronic pain. Use of regional techniques, either alone or in combination, may reduce opioid requirements and potentially the incidence of chronic pain[1] Thoracic paravertebral block and/or PECS block can provide effective analgesia and are usually combined with general anaesthesia for breast cancer surgery.

For some patients GA is undesirable due to various factors including patient choice, severe cardiovascular comorbidity, difficult airway. Combined thoracic paravertebral block with PECS 2 block is an effective alternative to general anaesthesia for high risk patients undergoing major breast surgeries.[2]

\section{Methods:}

We present a 77-year-old ASA 3 female who presented initially for right mastectomy and sentinel node biopsy and subsequently for Right axillary node clearance. She had Chemo-radiotherapy for laryngeal carcinoma in the past and had an unpleasant experience with awake fibreoptic intubation.

She was initially scheduled for right mastectomy and sentinel node clearance. A gas induction was attempted and resulted in failed intubation. Forty-eight hours later following discussion she was consented for thoracic paravertebral block combined with PECS block as mode of anaesthesia for the surgery. Patient was very keen to avoid general anaesthesia.

Intraoperatively following routine monitoring, she had $1 \mathrm{mg}$ midazolam and $25 \mathrm{mcg}$ of fentanyl as anxiolysis during regional block. USG paramedian approach thoracic paravertebral block was performed in the sitting position at two vertebral levels T 2-3 and T 4-5. $10 \mathrm{mls}$ of an equal mixture of 2\% lidocaine with 1:200000 epinephrine and $0.5 \%$ levo bupivacaine was administered at each level. Patient was positioned supine and USG PECS 2 block was performed with $10 \mathrm{mls}$ of $0.125 \%$ levo bupivacaine mixed with $5 \mathrm{mls}$ of $1 \%$ lidocaine with adrenaline and $10 \mathrm{mls}$ of normal saline. Patient had sedation during the procedure with Propofol TCI with effect site concentration titrated between 0.5 to $0.8 \mathrm{mcg} / \mathrm{ml}$. She was able to converse throughout surgery and maintained spontaneous ventilation. Supplemental oxygen was provided with optiflow and end tidal $\mathrm{CO} 2$ was monitored.
She subsequently was scheduled few months later for Right axillary node clearance and requested for the same anaesthetic as her previous breast surgery. Similar anaesthetic was provided with good outcome

\section{.Result:}

In both the occasions patient was very comfortable both during intraoperative and perioperative period. Her analgesic requirement in the postoperative period was minimal and tolerated well with regular paracetamol.

Patient allayed her fear of having an anaesthetic following her traumatic experience with previous general anaesthetics.

\section{Conclusions:}

Awake major breast surgeries can be successfully performed with combination of thoracic paravertebral and PECS 2 blocks. This has been demonstrated in our patient not once but twice.

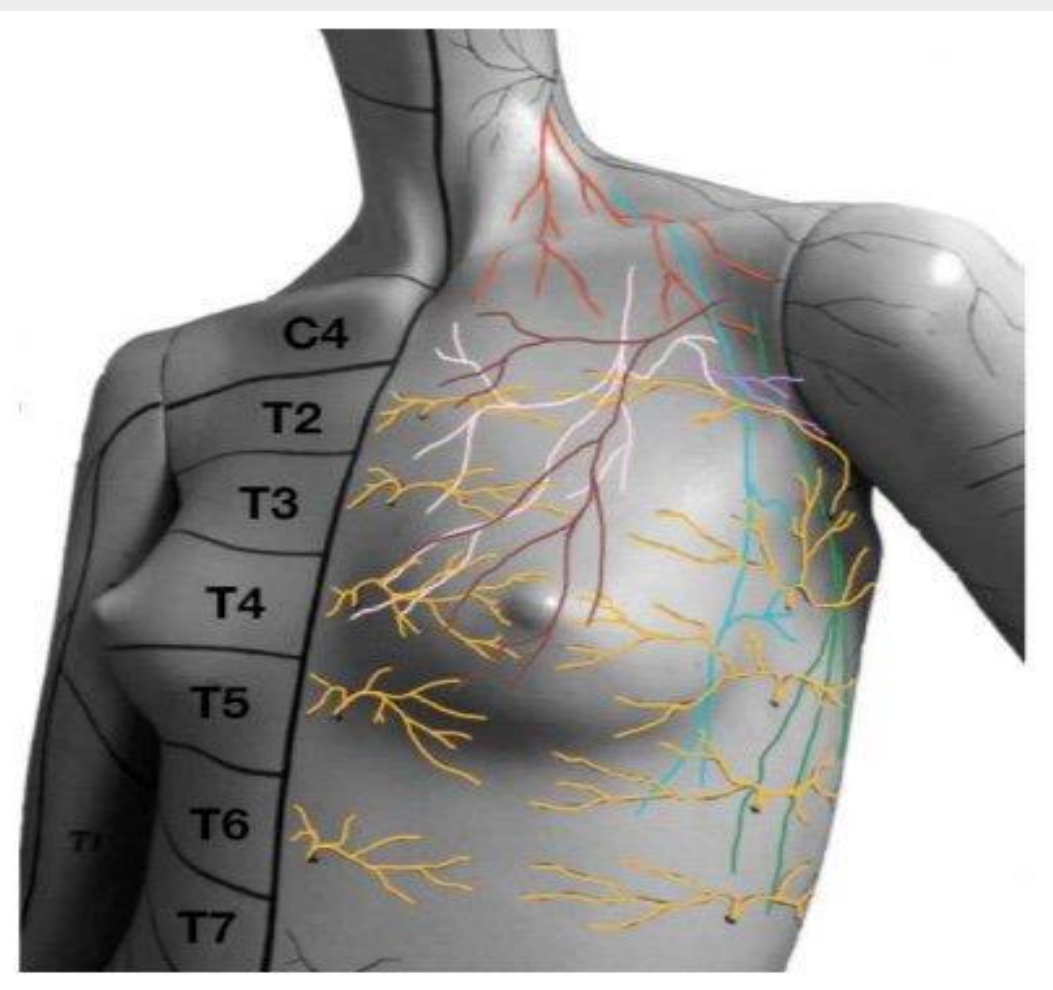

Figure 1 Innervation of the breast. Anterior and lateral branches of intercostal nerves (yellow); supraclavicular nerves (orange); lateral pectoral nerve (brown); medial pectoral nerve (pink); long thoracic nerve (blue); thoracodorsal nerve (green); intercostobrachial (purple).

\section{Reference:}

1, Heesen M, Klimek M, Rossaint R, Imberger G, Straube S. Paravertebral block and persistent postoperative pain afterbreast surgery: meta-analysis and trial sequential analysis.Anaesthesia 2016;71: 1471-81

2, Pawa.A et al Combined thoracic paravertebral and pectoral nerve blocks for breast surgery under sedation: a prospective observational case series. Anaesthesia 2018, 73: 438-443 metus. 